\title{
Propriedades farmacológicas do Sesquiterpeno a - Bisabolol: uma breve revisão
}

Pharmacological properties of a - Bisabolol Sesquiterpen: a brief review

Propiedades farmacológicas del Sesquiterpeno a - Bisabolol: una breve revisión

\author{
Elaine Roberta Leite de SOUZA ${ }^{1}$ \\ Nilvia Maria Lima GOMES ${ }^{\mathbf{A}}$
}

José Henrique de Araújo $\mathbf{C R U Z}^{1}$

Jessica Fernanda Delfino dos SANTOS ${ }^{1}$

Abrahão Alves de OLIVEIRA FILHO

${ }^{1}$ Curso de Graduação em Odontologia. Centro de Saúde e Tecnologia Rural, Universidade Federal de Campina Grande, UFCG 58708-110 Patos/PB, Brasil ${ }^{2}$ Professor(a) Doutor(a) do Curso de Graduação em Odontologia. Centro de Saúde e Tecnologia Rural, Universidade Federal de Campina Grande, UFCG 58708-110 Patos/PB, Brasil

\section{Resumo}

Introdução: O a-bisabolol é um álcool sesquiterpênico monocíclico amplamente utilizado em formulações cosméticas, o qual foi isolado de flores da camomila Objetivo: O objetivo do presente estudo foi realizar uma revisão de literatura sobre as propriedades farmacológicas do $\alpha$ bisabolol e sua aplicação como alternativa para o tratamento das mais diversas patologias que podem acometer o ser humano. Material e método: Foi realizada uma seleção de artigos científicos recuperados a partir das bases de dados: Lilacs, MEDLINE, BVS e Scielo, além de monografias que atenderam aos requisitos do tema abordado, apresentando informações confiáveis e publicados em bases de dados no período 2008 a 2018 com exceção de artigos clássicos que se apresentaram imprescindíveis ao presente estudo. Do total, 2.072 artigos foram obtidos e destes foram selecionados 28 artigos como amostra, que apresentaram a temática elencada para a pesquisa e que foram discutidos nas seguintes sessões: a) caracterização e estrutura química; b) potencial antimicrobano c) potencial anti-inflamatório; d) potencial gastoprotetor; e) potencial antineoplásico e antimetastático. Resultados: o $\alpha$-bisabolol demonstra um grande potencial e eficácia como agente antimicrobiano, gastroprotetor, anti-inflamatório, antineoplásico e antimetastático observados em estudos in vitro e in vivo. Tal substância amplamente conhecida e utilizada vem demonstrando resultados positivos e animadores para o tratamento das mais diversas afecções que acometem a saúde da população, tanto em sua forma original quanto seus derivados. Conclusão: Portanto, as reduzidas pesquisas, principalmente in vivo, reforçam a necessidade de se intensificar estudos sobre o assunto.

Descritores: Plantas Medicinais; Terpenos; Farmacologia; Fitoterapia.

\section{Abstract}

Introduction: A-bisabolol is a monocyclic sesquiterpene alcohol widely used in cosmetic formulations, which has been isolated from chamomile flowers. The objective of the present study was to conduct a literature review on the pharmacological properties of $\alpha$-bisabolol and its application as alternative for the treatment of the most diverse pathologies that can affect the human being. Material and method: A selection of scientific articles retrieved from the Lilacs, MEDLINE, BVS and Scielo databases was carried out, as well as monographs that met the requirements of the topic addressed, presenting reliable information published in databases in 2008 to 2018 with the exception of classic articles that were essential to the present study. Of the total, 2,072 articles were obtained and of these 28 articles were selected as a sample, which presented the theme listed for the research and which were discussed in the following sessions: a) characterization and chemical structure; b) antimicrobial potential c) anti-inflammatory potential; d) potential protective expenditure; e) antineoplastic and antimetastatic potential. Results: $\alpha$-bisabolol demonstrates great potential and efficacy as antimicrobial, gastroprotective, anti-inflammatory, antineoplastic and antimetastatic agents observed in in vitro and in vivo studies. This widely known and used substance has been demonstrating positive and encouraging results for the treatment of the most diverse conditions that affect the health of the population, both in its original form and its derivatives. Conclusion: Therefore, the reduced research, especially in vivo, reinforces the need to intensify studies on the subject.

Descriptors: Medicinal Plants; Terpenes; Pharmacology; Phytotherapy.

\section{Resumen}

El objetivo de este estudio fue realizar una revisión de literatura sobre las propiedades farmacológicas del $\alpha$-bisabolol y su aplicación como una de las principales causas de la aparición de la coliflor, alternativa para el tratamiento de las más diversas patologías que pueden acometer al ser humano. En el presente trabajo se analizaron los resultados obtenidos en el análisis de los resultados obtenidos en el análisis de los resultados obtenidos, a 2018 con excepción de artículos clásicos que se presentaron imprescindibles para el presente estudio. Del total, 2.072 artículos fueron obtenidos y de éstos se seleccionaron 28 artículos como muestra, que presentaron la temática elaborada para la investigación y que fueron discutidos en las siguientes sesiones: a) caracterización y estructura química; b) potencial antimicrobiano c) potencial antiinflamatorio; d) potencial de gasto; e) potencial antineoplásico y antimetastático. Resultados: el $\alpha$-bisabolol demuestra un gran potencial y eficacia como agente antimicrobiano, gastroprotector, anti-inflamatorio, antineoplásico y antimetastático observados en estudios in vitro e in vivo. Esta sustancia ampliamente conocida y utilizada viene demostrando resultados positivos y alentadores para el tratamiento de las más diversas afecciones que afectan la salud de la población, tanto en su forma original como en sus derivados. Conclusión: Por lo tanto, las reducidas investigaciones, principalmente in vivo, refuerzan la necesidad de intensificar estudios sobre el tema.

Descriptores: Plantas Medicinales; Terpenos; Farmacología; Fitoterapia.

INTRODUÇÃO

Desde o início da civilização humana, as plantas têm sido utilizadas com fins terapêuticos. $\mathrm{O}$ descobrimento de suas propriedades medicinais era meramente intuitivo ou, eventualmente, pela observação dos animais, que buscavam nas ervas o alívio para suas enfermidades ${ }^{1}$.

Com base nisso, os óleos e extratos de plantas há muito tempo têm servido de base para diversas aplicações na medicina, indústria farmacêuticas, de alimentos e cosméticos, contribuindo nos prazeres de sabores naturais e fragrâncias, além de possuírem várias propriedades farmacológicas, como, antinociceptiva e antiinflamatória ${ }^{2}$, antimicrobiana $^{3}$, antitumoral $^{4}$ e gastroprotetor ${ }^{5}$.

O a-bisabolol é um álcool sesquiterpênico monocíclico amplamente utilizado em formulações cosméticas, o qual foi isolado de flores da camomila (Matricaria chamomilla) ${ }^{6}$. Também conhecido como levomenol, está presente em uma diversidade de plantas, podendo ser obtido por hidrodestilação. Dentre as fontes deste óleo podemos citar a camomila alemã (Mattricaria chamomilla) e a sálvia (Salvia runcinata) que contém entre 50 e $90 \%$ de $\alpha$-bisabolol, 
respectivamente ${ }^{7}$ Q Quimicamente, o $\alpha$-bisabolol, também conhecido como alfa 4-dimetil-alfa-(4-metil3-pentenil)-3-ciclo-hexeno-1-metanol, com a fórmula química $\mathrm{C} 15 \mathrm{H} 26 \mathrm{O}$, tem fraco odor floral e adocicado. Ele é um líquido sem coloração, sua densidade é moderadamente baixa $(0,93 \mathrm{mg} / \mathrm{mL})$ e um ponto de ebulição de $153{ }^{\circ} \mathrm{C}$ a 12 Torr. Trata-se de uma substância praticamente insolúvel em água e muito solúvel em etanol ${ }^{8}$.

O $\alpha$-bisabolol vem sendo bastante utilizado pelas indústrias de medicamentos e cosméticos, devido ao seu comprovado poder anti-inflamatório. Ainda possui uso aclamado na indústria cosmética e nas farmácias de manipulação. De modo geral, os óleos essenciais extraídos de plantas podem apresentar efeito alelopático, pois se revelam como potentes inibidores da germinação de sementes e do desenvolvimento de diferentes espécies de plantas $^{6,9,10}$.

O objetivo do presente estudo foi realizar uma revisão de literatura sobre as propriedades farmacológicas do $\alpha$-bisabolol e sua aplicação como alternativa para o tratamento das mais diversas patologias que podem acometer o ser humano.

\section{MATERIAL E MÉTODO}

O presente estudo trata-se de uma revisão bibliográfica do tipo narrativa, no qual foi realizada uma seleção de artigos científicos recuperados a partir das bases de dados: Lilacs (Centro LatinoAmericano e do Caribe de Informação em Ciências da Saúde), MEDLINE (Medical Literature Analysis and Retrieval Sistem Online), BVS (Biblioteca Virtual em Saúde) e Scielo (Scientific Eletronic Library Online), além de monografias que atenderam aos requisitos do tema abordado, apresentando informações confiáveis e publicados em bases de dados no período 2008 a 2018 com exceção de artigos clássicos que se apresentaram imprescindíveis ao presente estudo.

A pesquisa foi realizada de 25 de maio a 01 de junho de 2018, nos idiomas português e inglês. A estratégia de busca utilizada para pesquisa nas bases de dados se deu pela utilização dos seguintes descritores isolados ou em combinação: "alfabisabolol" "camomila", "propriedades farmacológicas", "fitoterapia", “antimicrobiano", "anti-inflamatório", "antiviral", "antineoplásico", "antimetastático", obtendo-se um total de 2.072 artigos. Foram selecionados 28 artigos como amostra, que apresentaram a temática elencada para a pesquisa e que foram discutidos nas seguintes sessões: a) caracterização e estrutura química; b) potencial antimicrobiano c) potencial anti-inflamatório; d) potencial gastroprotetor e; e) potencial antineoplásico e antimetastático.

\section{RESULTADOS E DISCUSSÃO}

- Caracterização e estrutura química do $\alpha$-bisabolol
Os terpenos, também designados por terpenóides ou isoprenóides abrangem a maior e mais importante classe de substâncias derivadas de plantas, fungos e organismos marinhos ${ }^{11}$. Os compostos terpenicos classificam-se acordo com a sua unidade de isopreno (5C), monoterpenos (10C), sesquiterpenos (15C); diterpenos (20C); sesterpenos (25C); triterpenos (30C); tetraterpenos (40C) e polisoprenóides (nC) sendo os monoterpenos e os sesquiterpenos os constituintes de maior abundância $^{12}$.

Breitmaier $^{13}$ define os sesquiterpenos como sendo uma classe de substâncias estruturalmente formadas por três unidades de isopreno contendo, cada uma, um esqueleto com cinco carbonos. $\mathrm{O} \alpha-$ bisabolol é uma álcool sesquiterpênico monocíclico, lipofílico, volátil e de baixo peso molecular da classe dos bisabolanos.

O $\alpha$-bisabolol foi sintetizado pela primeira vez em 1951 a partir das flores de Matricaria chamomila, popularmente conhecida como Camomila, porém, pode ser obtido através da destilação direta de outros gêneros vegetais, como Peperomia e Vanillosmopsis ${ }^{14}$.

O $\alpha$-bisabolol pode ser encontrado na natureza na forma de 4 estereoisômeros: (-)- $\alpha$ bisabolol, (-)-api- $\alpha$-bisabolol, (+)- $\alpha$-bisabolol e (+)epi- $\alpha$-bisabolol, sendo que apenas sua forma $(-)-\alpha-$ bisabolol apresenta potencial farmacológico ${ }^{6}$.

\section{- Potencial Antimicrobiano}

A crescente resistência dos microrganismos tem levado os pesquisadores a buscar outras fontes para a produção de medicamentos que tragam a mesma eficácia, segurança e compatibilidade que os medicamentos sintéticos. Assim, as plantas medicinais juntamente com os óleos essenciais e seus compostos majoritários podem ser uma boa alternativa como fonte de matéria prima para a produção de novos medicamentos ${ }^{15}$.

Bezerra $^{5}$ analisou a atividade gastroprotetora e antimicrobiana do extrato seco de Matricaria chamomila (Camomila) e do $\alpha$-Bisabolol e seus possíveis mecanismos de ação. Para isso, foi utilizado o método de difusão em Agar para determinar o potencial antimicrobiano do Extrato seco de Matricaria camomila e do $\alpha$-bisabolol sobre cepas microbianas originárias da ATCC e de origem hospitalar (Staphylococcus aureus ATCC 6538, Candida albicans ATCC 10231, Klebsiella pneumoniae e Acinetobacter balmanii). Foi demonstrado que o extrato seco de Matricaria chamomila não conseguiu inibir o crescimento de nenhuma das cepas testadas e determinou apenas uma pequena inibição para a levedura $C$. albicans na concentração de $5 \mathrm{mg} / \mathrm{mL}$ enquanto que o $\alpha$-bisabolol inibiu o crescimento de $S$. aureus ATCC 6538, $C$. albicans ATCC 10231, Klebsiella pneumoniae e Acinetobacter balmanii até nas concentrações de 
0,0625, 0,0625, 4 e $4 \mathrm{mg} / \mathrm{mL}$, respectivamente. Foi visto também que a maior atividade antimicrobiana apresentada do $\alpha$-bisabolol foi sobre a bactéria Grampositiva $S$. aureus ATCC 6538, com uma Concentração Inibitória Mínima (CIM) de $1 \mathrm{mg} / \mathrm{mL}$ e para as demais cepas testadas, as CIM do Extrato e do $\alpha$-bisabolol foram maiores que $1 \mathrm{mg} / \mathrm{mL}$.

Um estudo publicado por Carcamo et al. ${ }^{16}$ buscou avaliar a efetividade antimicrobiana de um enxaguatório bucal a base de Matricaria chamomila, principal fonte de $\alpha$-bisabolol, em funcionários da Universidade de Desarrollo no Chile. Participaram do estudo 32 funcionários que foram divididos em 4 grupos: (1) pacientes que utilizaram o enxaguante a base do extrato de Matricaria chamomila; (2) pacientes que utilizaram soro fisiológico $0,9 \%$; (3) pacientes que utilizaram Clorexidina $0,12 \%$ (controle negativo) e (4) pacientes que não utilizaram nada (controle positivo). $\mathrm{O}$ pacientes dos grupos 1,2 e 3 foram orientados a fazer os bochechos por 1 minuto após a profilaxia feita pelo pesquisador e realizou-se o cultivo microbiológico em 8 tempos: (0) anamnese, ficha clínica e índice de Loe; (1) amostra após profilaxia profissional; (2) amostra após o uso do enxaguatório; (3) 30 minutos após o enxague; (4) 1 hora após enxague; (5) duas horas após o enxague; (6) 4 horas após o enxague; (7) 8 horas após o enxague e (8) novo índice de Loe (quantidade de placa bacteriana persente nas superfícies dentarias). Como resultados foi constatado que o Extrato de Matricaria chamomila, que contem de 50 a $90 \%$ de $\alpha$-bisabolol em sua composição foi eficaz como agente antimicrobiano, mantendo a sua ação por 8 horas, sendo que evidenciou uma diminuição da carga microbiana mais satisfatória nas primeiras 4 horas.

Rocha et al. $^{17}$ avaliaram a atividade antifúngica do óleo essencial de Vanillosmopsis arborea Baker e seu composto majoritário $\alpha$ bisabolol contra cepas de Candida albicans, Candida tropicallis, Aspergillus niger e Penicillium notatum. O óleo essencial de Vanillosmopsis arborea e o $\alpha$ bisabolol foram submetidos à avaliação da atividade antifúngica através do método de microdiluição para avaliar a Concentração Inibitória Mínima (CMI) e a atividade moduladora utilizando do óleo essencial e do $\alpha$-bisabolol frente ao cetoconazol e fluconazol. Como resultados obtidos, pode-se observar que tanto o óleo essencial de Vanillosmopsis arborea quanto o $\alpha$-bisabolol foram capazes de inibir o crescimento de todas as cepas fúngicas testadas. Quanto a atividade moduladora, foi visto que o óleo essencial não afetou a ação dos antifúngicos testados (cetoconazol e fluconazol), enquanto que o $\alpha$-bisabolol potencializou a ação do fluconazol frente à Penicillium notatum.

- Potencial Anti-inflamatório

Neville et al. ${ }^{18}$ e Ribeiro et al. ${ }^{19}$ definem o processo inflamatório como sendo uma resposta natural do organismo frente a invasão por um agente infeccioso ou a um dano tecidual sendo representada por um conjunto de reações locais e gerais do organismo representados pelos cinco sinais patognomônicos da inflamação que seriam a dor, rubor, calor, edema e perda de função.

$\mathrm{O}$ estudo realizado por Kim et $\mathrm{al}^{20}{ }^{20}$ se propuseram a investigar os efeitos anti-inflamatórios do $\alpha$-bisabolol e seus possíveis mecanismos de ação. Foi observado que $\alpha$-bisabolol pôde inibir a produção de óxido nítrico (NO) induzida por lipopolissacarídeos (LPS) e prostaglandina E (2) (PGE (2) em células RAW264.7. Além disso, a expressão dos genes da ciclooxigenase-2 (COX-2) e do óxido nítrico sintetase indutível (iNOS) foi reduzida. Para avaliar o mecanismo da propriedade anti-inflamatória do $\alpha$-bisabolol, os seus efeitos na atividade dos promotores AP-1 e NF-kB foram examinados. A ativação induzida por LPS dos promotores AP-1 e NF-kB foi significativamente reduzida pelo a-bisabolol. $\mathrm{O} \alpha$-bisabolol reduziu

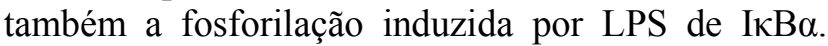
Além disso, enquanto a fosforilação induzida por LPS de ERK e p38 foi atenuada pelo $\alpha$-bisabolol, não foram observadas alterações significativas no nível de JNK fosforilada. Os resultados do estudo podem concluir que o $\alpha$-bisabolol exerce efeitos antiinflamatórios através da regulação negativa da expressão dos genes iNOS e COX-2 através da inibição da sinalização de NF-kB e AP-1 (ERK e p38).

Leite et al. ${ }^{9}$ buscaram examinar os efeitos anti-inflamatórios e antinociceptivos do $\alpha$-bisabolol (BISA). Para avaliar seu potencial anti-inflamatório o $\alpha$-bisabolol foi testado sob modelos agudos de dermatite induzida por óleo de Croton, ácido araquidônico, fenol e capsaicina em orelha de camundongo e seu potencial antinociceptivo foi testado sob dois modelos estabelecidos de ratinho de nocicepção visceral. Os ratinhos foram tratados oralmente com BISA, e as respostas comportamentais relacionadas com a dor à ciclofosfamida intraperitoneal ou a óleo de mostarda intracolónico foram avaliadass. Foi observado, sobre seu potencial anti-inflamatorio que o $\alpha$-bisabolol foi capaz de inibir a dermatite induzida por todos os agentes nocivos testados no estudo, com exceção da capsaicina e que, sobre seu potencial antinociceptivo, mostrou uma redução significativa da dor relacionada à dose administrada. Em conjunto, foi demonstrado que o $\alpha$ bisabolol pode ser um agente antinociceptivo e antiinflamatório visceral tópico.

- Potencial Gastroprotetor

As úlceras gástricas e duodenais caracterizam-se clinicamente pela presença de uma ou mais lesões erosivas, de origem multifatorial, que se estendem através da camada muscular à submucosa, ou ainda mais profundamente em 
detrimento da digestão ácido péptico dos tecidos. Dentre os fatores etiológicos que podem causar as lesões gástricas e duodenais, pode-se citar uma alimentação não saudável, estresse emocional, fatores genéticos e, principalmente, uso repetido e exagerado de AINEs e infecção por Helicobacter pylori ${ }^{21,22}$.

Acerca dos achados de Bezerra ${ }^{5}$ sobre o potencial gastroprotetor do $\alpha$ - bisabolol e do extrato seco das flores de Matricaria chamomila, foi visto que Extrato Seco nas doses 100, 200 e $400 \mathrm{mg} / \mathrm{Kg}$ introduzido oralmente, reduziu em 78,68 e $89 \%$, respectivamente $(\mathrm{p}<0,001)$ as lesões gástricas induzidas por etanol $(1,0 \mathrm{~mL} / \mathrm{animal})$ em ratos. $\mathrm{O} \alpha$ bisabolol também inibiu a formação de úlceras induzidas por etanol em doses menores e com ais eficácia que o Extrato seco (50 e $100 \mathrm{mg} / \mathrm{Kg})$, reduzindo em $87 \%$ e $96 \%$, respectivamente. O mecanismo de gastroproteção do $\alpha$ - bisabolol nas lesões gástrica induzidas por etanol em ratos foi avaliado na dose de $100 \mathrm{mg} / \mathrm{Kg}$. Para estabeler o mecanismo de ação do $\alpha$ - bisabolol foi feito um prétratamento com L-NAME e com indometacina (um inibidor das prostaglandinas) e outro pré-tratamento utilizando glibenclamida (inibidor dos canais $\mathrm{K}$ +ATP). Como resultados, pode-se observar que o LNAME e a indometacina não bloquearam efetivamente $o$ efeito gastroprotetor, mas a glibenclamida reduziu significativamente $(\mathrm{p}<0,05)$ o efeito gastroprotetor produzido pelo $\alpha$-bisabolol. $\mathrm{O}$ estudo concluiu, acerca dos efeitos gastroprotetores, que o $\alpha$-bisabolol tem a capacidade de diminuir as lesões da mucosa gástrica produzidas por etanol pela via de mecanismo de ativação dos canais $\mathrm{K}+\mathrm{ATP}$.

Duarte et al. ${ }^{23}$ realizaram um estudo in vivo, com uma amostra de 36 ratos pretendendo avaliar o efeito da camomila, principal fonte de $\alpha$ - bisabolol, sobre a cicatrização de úlceras que foram induzidas nas línguas de ratos limitadas à mucosa. A amostra foi dividida em dois grupos distintos. $\mathrm{O}$ primeiro grupo, composto pela metade da amostra da pesquisa recebeu aplicações de $12 \mathrm{em} 12$ horas de $0,02 \mathrm{~mL}$ de extrato de camomila a $10 \%$ durante 10 dias enquanto que o grupo controle não recebeu nenhum tratamento. Ao final do período do estudo foi visto que a camomila apresentou melhores resultados em relação à epitelialização e percentual de fibras colágenas. Porém a camomila não influenciou no grau da inflamação e nem no tamanho da lesão tecidual.

\section{- Potencial Antineoplásico e Antimetastático}

As neoplasias malignas podem ser definidas como em crescimento rápido e descontrolado de células que pode se disseminar por todos os tecidos e que tal crescimento não para após ser cessado o estimulo $^{24}$. Estando como a segunda causa de morte no Brasil, dados do Instituto Nacional do Câncer $(\text { INCA })^{25}$ sobre a incidência de câncer no Brasil no biênio 2016-2017 apontam o surgimento de $600 \mathrm{mil}$ novos casos de câncer nesse período, sendo o câncer de próstata nos homens e o de mama nas mulheres os com maior incidência e que em 2030, a carga global será de 21,4 milhões de casos novos de câncer e 13,2 milhões de mortes por câncer, em consequência do crescimento e do envelhecimento da população.

Entre os fatores de risco para o desenvolvimento do câncer estão o tabagismo, exposição prolongada ao sol, consumo excessivo de álcool além de fatores genéticos e ambientais (INCA, 2016-2017) $)^{25}$. Casarin et al. $^{26}$ revelam que para o tratamento de câncer as técnicas terapêuticas mais utilizadas são a quimioterapia, a radioterapia, a hormonioterapia, a imunoterapia e a intervenção cirúrgica. Porém mais recentemente se deu o uso de outras técnicas de tratamento, as chamadas práticas alternativas, sendo distinguida pela utilização de meios não tão tradicionais, entre os quais incluem os fitoterápicos e plantas medicinais.

O estudo realizado por Costarelli et al. ${ }^{4}$ objetivou investigar o papel desempenhado por duas doses de $\alpha$-bisabolol (via infusão intramamária) na indução e desenvolvimento de tumor mamário em camundongos transgênicos HER-2 / neu, bem como o efeito do a-bisabolol após ressecção cirúrgica tumoral. Os principais resultados obtidos mostram que (1) a dosagem ideal de $\alpha$-bisabolol é de $10 \mathrm{mg} /$ rato em vez de 3,6 $\mathrm{mg} /$ rato sem efeitos adversos (por exemplo, alopecia); (2) o número de massas tumorais palpáveis diminuiu em camundongos tratados com $10 \mathrm{mg} /$ rato de $\alpha$-bisabolol; (3) ratos após ressecção cirúrgica do tumor primário e tratamento com $\alpha$-bisabolol (10 mg) são livres de tumor por mais semanas, após o tratamento cirúrgico; Fois visto tambem que apos o tratamento $\operatorname{com} \alpha$ bisabolol alguns genes implicados nos mecanismos de carcinogênese (NF-kBia, Map2k, Mapk14 e HER2 / neu), o processo de angiogênese (Fgf) e a inibição da apoptose (Birc5) são regulados de forma diferente e a citotoxicidade das células NK aumenta nos ratos tratados, especialmente após a remoção da primeira massa tumoral.

O estudo de Seki et al. ${ }^{27}$ comprovaram que o $\alpha$-bisabolol, tem efeitos antitumorais contra o câncer de pâncreas. Foi visto queo a-bisabolol induziu uma diminuição na proliferação celular e viabilidade em linhas celulares de câncer de pâncreas (KLM1, KP4, Panc1, MIA Paca2), mas não em células epiteliais pancreáticas (ACBRI515). Além disso, o tratamento com $\alpha$-bisabolol induziu a superexpressão da resposta de crescimento precoce-1 (EGR1), enquanto que o EAR1 de siRNA diminuiu a morte celular induzida por $\alpha$-bisabolol de células KLM1. O crescimento tumoral nos modelos de ratos nus xenotransplante subcutâneo e peritoneal foi significativamente inibido pela administração intragástrica de $1000 \mathrm{mg} / \mathrm{kg}$ de $\alpha$-bisabolol, uma vez por semana durante três semanas. Os resultados indicam que o $\alpha$-bisabolol 
pode ser uma nova opção terapêutica para o tratamento do câncer de pâncreas.

Outro estudo sobre o potencial efeito antineoplásico do $\alpha$-bisabolol para tratamento de câncer de pâncreas desenvolvido por Murata et al. ${ }^{28}$ (2017) buscou desenvolver derivados de $\alpha$-bisabolol que sejam mais potentes do que o composto original e podem ser clinicamente úteis contra o câncer de pâncreas. Em primeiro lugar, foram desenhados e sintetizados 22 derivados de a-bisabolol. Os derivados de $\alpha$-Bisabolol 4 e 5 tiveram efeitos inibitórios mais potentes sobre a proliferação de células de câncer de pâncreas do que o a-bisabolol. Em seguida, foram desenhados e sintetizados 15 derivados adicionais de bisabolol com base na estrutura dos derivados de a-bisabolol 4 e 5. Entre eles, o derivado de $\alpha$-bisabolol 5 teve o efeito inibidor mais forte na proliferação. Este novo composto reduziu a proliferação de várias linhas celulares de câncer de pâncreas, como KLM1, Panc1 e KP4. Além disso, o composto induziu níveis mais elevados de apoptose em linhas celulares de câncer de pâncreas do que o $\alpha$-bisabolol. O derivado de $\alpha$ Bisabolol 5 inibiu o crescimento do tumor de xenoenxerto e reduziu a disseminação do câncer de pâncreas para os nódulos peritoneais. O composto suprimiu fortemente a expressão de AKT nos nódulos peritoneais. Os dados apresentados no estudo indicam que o derivado de a-bisabolol 5 efetivamente impede a progressão do câncer de pâncreas através da inibição de AKT.

\section{CONCLUSÃO}

$\mathrm{O} \alpha$-bisabolol demonstra um grande potencial e eficácia como agente antimicrobiano, gastroprotetor, anti-inflamatório, antineoplásico e antimetastático observados em estudos in vitro e in vivo. Tal substância amplamente conhecida e utilizada vem demonstrando resultados positivos e animadores para o tratamento das mais diversas afecções que acometem a saúde da população, tanto em sua forma original quanto seus derivados. Porem, o reduzido número de pesquisas, principalmente in vivo, reforça a necessidade de se intensificar os estudos sobre o assunto.

\section{REFERÊNCIAS}

1. Marques THC, Santos PS, Melo CHS, Carvalho RBF, Lima LS, David JM, et al. Atividade anticolinesterásica e perfil químico de uma fração cromatográfica ativa do extrato etanólico das flores Bellis perennis L. (Asteraceae). Qim. nova. 2013;36:549-53.

2. Sousa OV, Soares Júnior DT, Del-Vechio G, Mattosinhos RG, Gattass CR, Kaplan MAC. Atividades antinociceptiva e antiinflamatória do óleo essencial de cascas de Duguetia lanceolata St. Hil., Annonaceae. Rev. bras. farmacogn. 2004;14:11-14.
3. Valeriano C, Piccoli RH, Cardoso MG, Alves E. Atividade antimicrobiana de óleos essenciais em bactérias patogênicas de origem alimentar. Rev. bras. plantas med. 2012;14:57-67.

4. Costareli A, Malavolta M, Giacconi R, Cipriano C, Gasparini N, Tesei S, et al. In Vivo Effect of $\alpha$ Bisabolol, a Nontoxic Sesquiterpene Alcohol, on the Induction of Spontaneous Mammary Tumors in HER-2/neu Transgenic Mice. Oncology Research Featuring Preclinical and Clinical Cancer Therapeutics. 2009;18:409-18.

5. Bezerra SB. Atividade gastroprotetora e antimicrobiana do extrato seco de Matricaria recutitia (camomila) e do alfa-bisabolol: possíveis mecanismos de ação [dissertação.] Fortaleza (CE). Faculdade de Medicina, Universidade Federal do Ceará; 2009.

6. Kamatou PPG, Viljoen MAA. Review of the Application and Pharmacological Proprieties of aBisabolol and a-Bisabolol-Rich Oils, Jounal. American Oil Chermical Society. 2010;87:1-7.

7. Viljoen AM, Gono-Bwalya AB, Kamatou GPP, Bașer KHC, Demirci B. The essential oil composition and chemotaxonomyof Salviastenophylla and its allies S. repens and S. runcinata. J Essent Oil Res. 2006;18:37-45.

8. Madhavan BN. Final report on the safety assessment of bisabolol. Int. j. toxicol. 1999;18:33-40.

9. Leite GO, Leite LH, Sampaio RS, Araruna MK, Menezes IR, Costa JG, et al. (-)- $\alpha$-bisabolol atenuantes visceral nociception inflammation in mice. Fitoterapia. 2010;82:208-11.

10. Souza Filho APS, Guilhon GMSP, Santos LS. Metodologias empregadas em estudos de avaliação da atividade alelopática em condições de laboratório -Revisão crítica. Planta Daninha. 2010;28:689-97.

11. Niederbacher B, Winkler JB, Schnitzler JP. Volatile organic compouds as non-invasive markers for plant phenotyping. J Exp Bot. 2015;66:5403-16.

12. Murakamy A, Tanaka T, Lee JY, Surh YJ, Kim HW, Kawabata K, et al. Zerumbone, a sequisterpenein subtropical ginger,suppresses skintumor initiation and promotion stages in ICP mice. Int. J Cancer. 2004;110:481-90.

13. Breitmaier E. Terpenes: importance, general structure na biosynthesis. Terpenes. 2006;1:19.

14. Fernandes MYSD. Efeito neuroprotetor do $\alpha$ bisabolol em camundongos submetidos á isquemia cerebral focal permanente [dissertação]. Fortaleza (CE): Faculdade de Medicina, Universidade Federal do Ceará; 2015.

15. Fernandes Júnior A, Silva GS, Barbosa LN, Alves FCB, Andrade BFMT, Albano M, et al. Medicinal Plants from the Brazilian Savanna with Antibacterial Properties. European Journal of 
Medicinal Plants. 2014;4:1-13.

16. Carcamo OV, Oliva, MP, Gonzalez CP. Efectividad Antimicrobiana del Colutorio de Matricaria recutita, en Funcionarios de la Facultad de Odontología de la Universidad del Desarrollo, Chile. Int. J. Odontostomat. 2011;5:179-84.

17. Rocha JE, Saraiva CRN, Santos PEV, Colares AV. Avaliação da atividade antifúngica do óleo essencial de Vanillosmopsis arborea (Asteraceae) baker e seu composto majoritário - $\alpha$-bisabolol. 2015.

18. Neville BW, Damm DD, Allen CM, Bouquot JE. Patologia Oral e Maxilofacial. Edição (3a). Rio de Janeiro: Elsevier; 2009.

19. Ribeiro BP, Pereira WS, Sousa AIP, Guerra RNM, Nascimento FRF. Alteração no perfil bioquímico induzido por reação inflamatória granulomatosa em camundondos. Rev. Ciênc. Saúde. 2010;12:73-9.

20. Kim S, Jung E, Kim JH, Park YH, Lee J, Park D. Inhibitory effects of (-)- $\alpha$-bisabolol on LPSinduced inflammatury response in RAW264.7 macrophages. Food chem. toxicol. 2011;20.

21. Jain KS, et al. Recent advances in proton pump inhibitors and management of acid-peptic disorders. Bioorg. med. chem. 2007;15:1181-205.

22. Musumba C, Pritchard DM, Pirmohamed M. Review article: cellular and molecular mechanisms of NSAID-induced peptic ulcers. Aliment Pharmacology and Therapeutis. 2009;30:517-31.

23. Duarte CME, et al. Effects of Chamomilla recutita (L.) on oral wound healing in rats. Med. oral patol. oral cir. Bucal. 2011;16:716-21.

24. Filho AAO, Leite WLO. Avaliação farmacológica de produtos naturais no combate ao câncer. Revista interdisciplinar em saúde. 2014:192-211.

25. INSTITUTO NACIONAL DO CÂNCER [INCA]. Estimativa 2016. Incidência de Câncer no Brasil. Disponível em: http://www.inca.gov.br/estimativa/2016/.

26. Casarin ST, Heck RM, Schwartz E. O uso de práticas terapêuticas alternativas, sob a ótica do paciente oncológico e sua família. Fam. Saúde Desenv. 2005;7:24-31.

27. Seki T, Kokuryo T, Yokoyama Y, Suzuki $\mathrm{H}$, Itatsu K, Nakagawa A, et al. Antitumor effects of $\alpha$-bisabolol against pancreatic câncer. Cancer Sci. 2011;102:2199-205.

28. Murata Y, Kokuryo T, Yokoyama Y, Yamaguchi J, Miwa T, Shibuya M, et al. The Anticancer Effects of Novel $\alpha$-Bisabolol Derivatives Against Pancreatic Cancer. Anticancer Res. 2017;37:589-98.

\section{CONFLITO DE INTERESSES}

Os autores declaram não haver conflitos de interesse.

\section{AUTOR PARA CORRESPONDÊNCIA}

\section{Elaine Roberta Leite de Souza}

Rua José Flósculos, número 156, bairro Frei Damião

58.600-000 Santa Luzia/PB.

Telefone: (83) 99944-1733.

E-mail: elaine_roberta5@hotmail.com
Submetido em 07/08/2018

Aceito em 12/03/2019 